\title{
Symmetry-breaking instability in a prototypical driven granular gas
}

\author{
Evgeniy Khain and Baruch Meerson \\ Racah Institute of Physics, Hebrew University of Jerusalem, Jerusalem 91904, Israel
}

\begin{abstract}
Symmetry-breaking instability of a laterally uniform granular cluster (strip state) in a prototypical driven granular gas is investigated. The system consists of smooth hard disks in a two-dimensional box, colliding inelastically with each other and driven, at zero gravity, by a "thermal" wall. The limit of nearly elastic particle collisions is considered, and granular hydrodynamics with the JenkinsRichman constitutive relations is employed. The hydrodynamic problem is completely described by two scaled parameters and the aspect ratio of the box. Marginal stability analysis predicts a spontaneous symmetry breaking instability of the strip state, similar to that predicted recently for a different set of constitutive relations. If the system is big enough, the marginal stability curve becomes independent of the details of the boundary condition at the driving wall. In this regime, the density perturbation is exponentially localized at the elastic wall opposite to the thermal wall. The short- and long-wavelength asymptotics of the marginal stability curves are obtained analytically in the dilute limit. The physics of the symmetry-breaking instability is discussed.
\end{abstract}

PACS numbers: 45.70.Qj

\section{INTRODUCTION}

Granular materials play an important role in industrial applications, geophysics and astrophysics. They are also of a great general interest to physicists, as each of the "phases" of granular materials: solid, liquid and gas have unusual properties that distinguish them from their classic (atomic) counterparts [1, 2]. We will consider granular gas (or rapid granular flow) and focus on a variant of clustering instability, a striking tendency of granular gases to form dense clusters. Clustering instability was first discussed in the context of a freely "cooling" granular gas [4, 5, 6]. Following these works, related clustering phenomena were investigated in driven granular gases as well, both in experiments [7, 8] and in particle simulations [9, 10, 11].

Granular clustering results from energy losses by inelastic collisions, and it is a manifestation of thermal condensation instability, also encountered in other fields, for example in gases and plasmas that cool by their own radiation [12]. Since the discovery of the clustering instability, the validity of granular hydrodynamics (see Ref. [13] for a review) has been under scrutiny [3]. In contrast to the clustering in a freely "cooling" granular gas, where one deals with a complex time-dependent process, steady states are achievable in driven granular systems. One of the simplest settings of this type is driving the granulate by a side wall at a zero gravity. Therefore, an ensemble of inelastically colliding hard spheres, confined in a box and driven by one or two "thermal" walls has served as a prototypical driven granular system [7, 9, 10, 14, 15, 16, 17. Steady states of this system have served as test beds for granular hydrodynamics and its violations. The first analysis of this system in the physical literature was performed, in one dimension, by Kadanoff et al. [9]. The nearly elastic particles were constrained to move on a straight line with energy input from the boundaries. Par- ticle simulations [9] showed that, for typical initial conditions, the system evolves to a state where the particles are separated into two groups. Almost all particles form a cluster in a small region of space, where they move with very small velocities, while a very few remaining particles move with high velocities. Clearly, this steady state cannot be described by granular hydrodynamics (actually, by any coarse-grained theory). Therefore, the results of Kadanoff et al. [9] brought into question the validity of granular hydrodynamics in general.

This question was addressed in two subsequent theoretical works [10, 11] that dealt with similar systems in two dimensions. Esipov and Pöschel [11] investigated an ensemble of nearly elastic hard disks in a circular box with the circumference serving as thermal wall. Grossman et al. [10] considered a rectangular box, one side of which served as thermal wall. Particle simulations [10, 11] showed granular clusters: dense and "cold" regions of granulate developing away from the thermal wall. In terms of thecoarse-grained particle density, these steady-state clusters had simple shapes: azimuthally uniform (circular state) [11] and laterally uniform (strip state) [10]. Grossman et al. also showed that, for nearly elastic collisions, the strip state is describable by a steady-state solution of granular hydrodynamic equations. The empiric constitutive relations suggested by Grossman et al. used simple interpolations between the low-density limit, where the constitutive relations are derivable systematically [18], and high-density limit where, close to the dense packing, free volume arguments can be used.

The results of Refs. [10, 11] showed that the anomaly observed in the one-dimensional setting [9] does not persist in higher dimensions. Clustered states qualitatively similar to those of Grossman et al. were observed in experiment of Kudrolli et al. [7] who investigated a system of spherical particles in a box, rolling on a smooth sur- 
face and driven by a rapidly vibrating side wall. The number of particles served as the control parameter in Ref. [7]. A dense cluster of the strip type was observed when the number of particles was big enough, in much the same way as in Ref. [10]. The basic physics of the strip state is simple and can be explained by the following hydrodynamic argument. Because of the inelastic collisions the particle random motion slows down (that is, the granular temperature decreases) with the increase of the distance from the driving wall. To maintain the momentum balance, the granular density should increase with this distance. When the total number of particles is big enough (the rest of parameters being the same), the density contrast becomes large, and the enhanced density region away from the driving wall is observed as the strip state.

The prototypical system exhibits many interesting phenomena of non-hydrodynamic nature. These include inelastic collapse [11, 15], possible lack of scale separation [10], non-Gaussianity in the particle velocity distribution [10, 16], normal stress difference and pressure nonuniformities 14, etc. For nearly elastic collisions, however, granular hydrodynamics was shown to yield an accurate quantitative description in the dilute limit [14], and reasonably accurate description for moderate and high granular densities [10]. Of course, the nearly elastic limit is quite restrictive for most of realistic granular flows. Still, this limit is conceptually important just because granular hydrodynamics can be used there. Granular hydrodynamics has a great predictive power and helps to identify important collective phenomena (shear flows and vortices, shocks, different modes of clustering flows etc.) that are difficult, if not impossible, to identify and predict in the language of individual particles. Once identified, these phenomena can then be investigated in experiment and simulations in more general (not necessarily hydrodynamic) formulations.

Therefore, granular hydrodynamics provide a leadingorder approach to a big ensemble of nearly-elastically colliding grains. This approach has been taken recently by Livne et al. 177 who employed granular hydrodynamics for a stability analysis of the strip state described above. The analysis revealed a spontaneous symmetry-breaking instability of the strip state with respect to perturbations along the strip. Well within the instability region, the clustered states with broken symmetry, found by a numerical solution of the steady state hydrodynamic equation, are strongly localized in the lateral direction: most of the particles are located in dense "islands" [17. These results indicate that the prototypical system can show a non-trivial behavior even in the leading-order, hydrodynamic limit. Indeed, this systems can be put into the list of pattern-forming systems far from equilibrium [19].

The present work focuses on a more detailed stability analysis of this system. Our first objective is to check to what extent the symmetry-breaking instability pre- dicted in Ref. [17] is sensitive to the constitutive relations. Livne et al. 177 employed the empiric relations suggested by Grossman et al. 10. Here we shall use the better known Jenkins-Richman (JR) relations [20. While the relations of Grossman et al. are more accurate for high densities (even including those close to the dense packing limit), the JR relations should work better at low and intermediate densities. We shall see, however, that the marginal stability curves, obtained with these two sets of relations, are not much different from each other. This implies that the symmetry-breaking instability is robust. Our second objective is to get more insight into the marginal stability problem and, where possible, to obtain analytic results. We shall show that the marginal problem is equivalent to an eigenvalue problem of quantum mechanics. An important finding here is a universal behavior of the marginal stability curves in the limit when the density perturbations are strongly localized at the elastic wall opposite to the thermal wall. In the dilute limit, we obtain analytically the short- and longwavelength asymptotics of the marginal stability curves and density eigenfunctions. We also give the physical interpretation to the symmetry-breaking instability and to the density borders of the instability region.

The rest of the paper is organized as follows. In Sec. 2 we formulate the model and briefly describe the strip state: the simplest steady state of the prototypical system. Section 3 presents marginal stability analysis of the strip state and compares the results obtained for two different sets of constitutive relations. More results on marginal stability, including some analytic results in the dilute limit, are presented in Sec. 4. Section 5 includes a discussion and summary.

\section{PROTOTYPICAL SYSTEM AND STRIP STATE}

The prototypical driven granular system in two dimensions include inelastically colliding hard disks of diameter $d$ and mass $m=1$, moving in a box with dimensions $L_{x} \times L_{y}$. The gravity force is zero. Collisions of disks with the walls $x=0, y=0$ and $y=L_{y}$ are assumed elastic. The wall $x=L_{x}$ is "thermal" wall: upon collision a particle is assigned a random velocity taken from a Gaussian ensemble with temperature $T_{0}$. Energy input at the thermal wall balances the energy dissipation due to inter-particle collisions, so the system can reach a steady state. We shall parameterize the inelasticity of the particle collisions by a constant normal restitution coefficient $r$ and work in the nearly elastic limit: $1-r^{2} \ll 1$. In this limit, the Navier-Stokes granular hydrodynamics is expected to be sufficiently accurate in a system with a big number of particles and small Knudsen number. The possible steady states of the system are described by the 
steady state versions of the momentum and energy balance equations:

$$
p=\text { const } \text { and } \nabla \cdot(\kappa \nabla T)=I,
$$

where $p$ is the granular pressure, $T$ is the granular temperature, $\kappa$ is the thermal conductivity and $I$ is the rate of energy losses by collisions. We assume that the number density $n$ is not too big: $n / n_{c} \leq 0.5$, where $n_{c}=2 /\left(\sqrt{3} d^{2}\right)$ is the (hexagonal) dense packing density. This assumption enables us to employ the constitutive relations derived by Jenkins and Richman [20]. For the steady state problem, the required constitutive relations include the equation of state $p=p(n, T)$ and relations for $\kappa$ and $I$ in terms of $n$ and $T$.

Let us introduce scaled coordinates: $\mathbf{r} / L_{x} \rightarrow \mathbf{r}$. In the new coordinates the box dimensions are $1 \times \Delta$, where $\Delta=$ $L_{y} / L_{x}$ is the aspect ratio. Introducing the normalized inverse density $z(x, y)=n_{c} / n(x, y)$, one can rewrite the energy balance equation in Eq. (1) in terms of $z(x, y)$ :

$$
\nabla \cdot(F(z) \nabla z)=\eta Q(z)
$$

where $F(z)=A(z) B(z)$,

$$
\begin{aligned}
& A(z)=\frac{G\left[1+\frac{9 \pi}{16}\left(1+\frac{2}{3 G}\right)^{2}\right]}{z^{1 / 2}(1+2 G)^{5 / 2}}, \\
& B(z)=1+2 G+\frac{\pi}{\sqrt{3}} \frac{z\left(z+\frac{\pi}{16 \sqrt{3}}\right)}{\left(z-\frac{\pi}{2 \sqrt{3}}\right)^{3}}, \\
& Q(z)=\frac{6}{\pi} \frac{z^{1 / 2} G}{(1+2 G)^{3 / 2}} \\
& G(z)=\frac{\pi}{2 \sqrt{3}} \frac{z-\frac{7 \pi}{32 \sqrt{3}}}{\left(z-\frac{\pi}{2 \sqrt{3}}\right)^{2}}
\end{aligned}
$$

and $\eta=(2 \pi / 3)(1-r)\left(L_{x} / d\right)^{2}$. Notice that, for an arbitrary small but finite inelasticity $1-r$, the dimensionless parameter $\eta$ can be made arbitrary large, if the system size $L_{x}$ is large enough. The parameter $\eta$ differs from the parameter $\mathcal{L}$ used by Livne et al. [17] only by a numerical factor of order unity. Of most interest are regimes where $\eta \gg 1$, see below.

The boundary conditions for Eq. (2) are determined by the properties of the particle-wall interactions. At the elastic walls $x=0, y=0$ and $y=\Delta$ we should prescribe a zero normal component of the heat flux. In terms of the inverse density $z$ we have $\nabla_{n} z=0$ at these three walls. Here index $n$ denotes the gradient component normal to the wall. The constant temperature at the "thermal" wall $x=1$ yields the condition $\partial z(x=1, y) / \partial y=0$. To make the formulation of the problem complete, one more condition is needed. In experiment or particle simulations, the number of particles $N$ is fixed. This yields a normalization condition:

$$
\frac{1}{\Delta} \int_{0}^{1} \int_{0}^{\Delta} \frac{d x d y}{z(x, y)}=f
$$

where $f=\langle n\rangle / n_{c}$ is the area fraction of the grains and $\langle n\rangle=N /\left(L_{x} L_{y}\right)$ is the average number density of the grains.

Equations (2)-(位) and the boundary conditions make a complete set. One can see that the governing parameters of this system are the scaled parameters $\eta, f$ and $\Delta$. If the system is infinite in the $y$-direction, only two governing parameters: $\eta$ and $f$ remain. Notice that the steady-state density distributions are independent of the wall temperature $T_{0}$, in contrast to the similar problem with gravity, where the gravity acceleration, combined with $T_{0}$ and the (finite) system size in the direction of gravity, would make an additional governing parameter.

The laterally uniform steady state (strip state) corresponds to the one-dimensional ( $y$-independent) solution $z=Z(x)$. It is described by the equations

$$
\begin{aligned}
\left(F Z^{\prime}\right)^{\prime}=\eta Q,\left.Z^{\prime}\right|_{x=0} & =0, \\
\text { and } \int_{0}^{1} Z^{-1}(x) d x & =f,
\end{aligned}
$$

where primes stand for the $x$ derivatives. For the strip state, the boundary condition at the wall $x=1$ drops out. This implies, in particular, that the density profile of the strip state is independent of the exact nature of the driving wall (thermal or vibrating wall) [21]. This degeneracy of the strip state is caused by the character of particle interaction: the hard-core potential does not introduce any characteristic energy [11]. Notice that, instead of prescribing the grain area fraction $f$, one can prescribe the inverse density $Z=Z_{0}$ at $x=0$. This condition, combined with the no-flux condition at $x=0$ defines a Cauchy problem for $Z(x)$. Solving the Cauchy problem, one can then compute, from the last equation in Eq. (5), the respective value of $f$. At fixed $\eta$, there is a one-to-one correspondence between $Z_{0}$ and $f$. Therefore, an alternative parameterization of the strip state is given by the scaled numbers $\eta$ and $Z_{0}$. We shall see below that the same property keeps (and can be conveniently used) in the marginal stability problem. Figure 1 shows a typical example of the scaled density profile $n(x) / n_{c}$ of the strip state obtained by solving Eqs. (5) numerically.

\section{INSTABILITY OF THE STRIP STATE: MARGINAL STABILITY CURVES}

In general, the strip state is only one of the possible solutions of Eq. (2). Because of its nonlinearity, Eq. (2) may have additional solutions satisfying the same boundary conditions. When exist, these additional solutions are truly two-dimensional: the translational symmetry along $y$ is broken. An important class of these solutions bifurcate supercritically from the strip state 17 . Therefore, close to the bifurcation point, these solutions can be found by linearizing Eq. (2) around the strip state. 


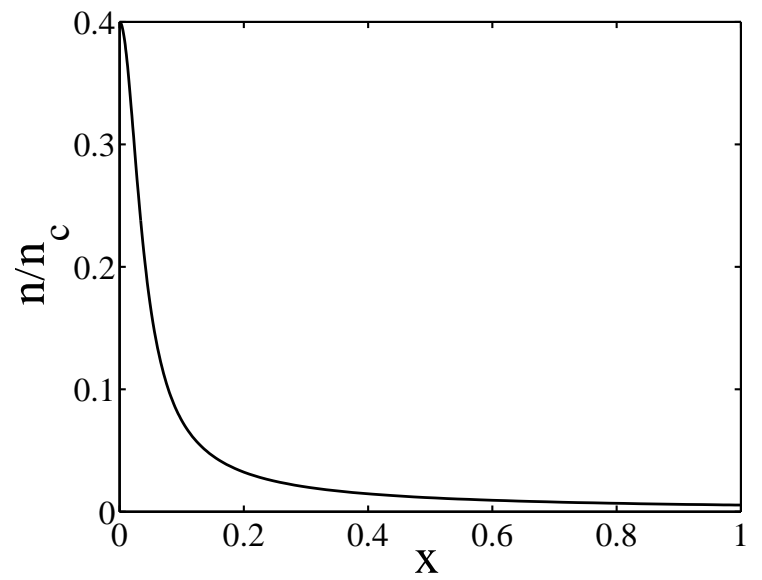

FIG. 1: An example of the scaled density profile $n(x) / n_{c}$ of the strip state for the JR constitutive relations. The governing parameters are $\eta=10^{4}$ and $f=0.0342$.

A similar analysis was performed in Ref. [17] for the constitutive relations of Grossman et al. 10]. In the framework of a time-dependent hydrodynamic formulation, this analysis corresponds to marginal stability analysis of the strip state with respect to small perturbations along the strip [17].

Substituting $z(x, y)=Z(x)+\psi_{k}(x) \cos k y$ and linearizing Eq. (2) with respect to the small correction $\psi_{k}(x) \cos k y$, we obtain:

$$
\phi^{\prime \prime}-\left(\frac{\eta Q_{Z}}{F}+k^{2}\right) \phi=0 .
$$

Here $\phi(x)=F(x) \psi_{k}(x)$, functions $F$ and $Q$ are evaluated at $z=Z(x)$, and subscript $Z$ means the $z$ derivative evaluated at $z=Z(x)$. The boundary conditions are

$$
\phi^{\prime}(x=0)=0 \text { and } \phi(x=1)=0 .
$$

Equation (6) coincides with the Schrödinger equation for an even wave function $\phi(x)$ of a particle in the potential well

$$
U(x)= \begin{cases}\frac{\eta Q_{Z}}{F} & \text { if }|x|<1, \\ +\infty & \text { otherwise }\end{cases}
$$

The quantity $-k^{2}$ serves as the energy eigenvalue. The energy levels in the potential (8) are always discrete, and there is an infinite number of them. However, as the wave number $k$ should be real, only negative or zero energy levels are admissible. At fixed values of $\eta$ and $f$, the potential (8) admits at most one such energy level. The absence of negative energy levels implies that, in the vicinity of the strip state, there are no steady states different from it. The presence of a negative energy level corresponds to a "weakly two-dimensional" steady state, bifurcating from the strip state. We shall exploit the quantum-mechanical analogy more fully in Sec. 4. Here we report some numerical results. Figure 2 shows the marginal stability curves: the curves $k=k(f)$ at different values of $\eta$, computed numerically. In these computations, the parameter $\eta$ was taken large enough. The strip state is unstable below the respective curve and stable above the curve. Notice that, at fixed $\eta$, the instability is possible only within a finite interval of $f$ : $f_{1}(\eta)<f<f_{2}(\eta)$. The same property was reported in Ref. [17] for another set of constitutive relations. We shall give a physical explanation to this finding in Sec. 4. Notice (see also Ref. [17) that, at large $\eta$, the highdensity stability border $f_{2}$ is quite small. The curves in Fig. 2 are actually plotted in scaled coordinates: $k \eta^{-1 / 2}$ versus $f \eta^{1 / 2}$. It can be seen that, in the scaled coordinates, all the curves exit from the same point of the horizontal axis $f \eta^{1 / 2}$. In addition, the maxima of all the curves are equal. These observations will be also explained in Sec. 4.

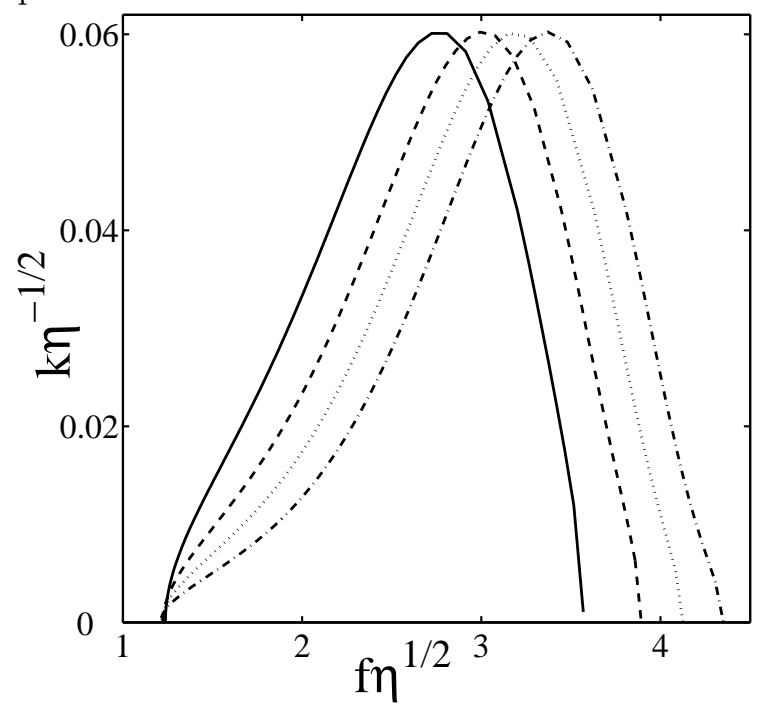

FIG. 2: Marginal stability curves for different values of $\eta$, plotted in scaled coordinates: $k \eta^{-1 / 2}$ versus $f \eta^{1 / 2}$. For a fixed $\eta$ the strip state is stable above the respective curve and unstable below the curve. The values of $\eta$ are: $10^{4}$ (solid line), $2.5 \cdot 10^{4}$ (dashed line), $5 \cdot 10^{4}$ (dotted line) and $10^{5}$ (dash-dot line).

If the system is infinite in the lateral direction, $\Delta=\infty$, while $\eta$ and $f$ are fixed, a whole continuum spectrum of wave numbers between $k=0$ and $k=k(\eta, f)$ is unstable. Both in experiment, and in numerical simulations $\Delta$ is finite. In this case $k$ becomes discrete because of the boundary conditions: $k=m \pi / \Delta$, where $m=1,2, \ldots$. For each $m$ we can find the critical value of the aspect ratio $\Delta$ (let us call it $\Delta_{m}$ ) such that for $\Delta>\Delta_{m}$ the strip state looses stability with respect to the $m$-mode. Obviously, $\Delta_{m}=m \Delta_{1}$, and $\Delta_{1}$ is the lowest critical value for the symmetry-breaking instability. Figure 3 shows $\Delta_{1}$ as a function of $f$ for different values of $\eta$. For fixed $\eta$ and $f$, the strip state is unstable above the curve. It is seen from Fig. 3 that, in order to observe the symmetry-breaking instability, one does not need to 
work with very large aspect ratios $\Delta$ : it is sufficient if the system is big enough, so that parameter $\eta$ is sufficiently large.

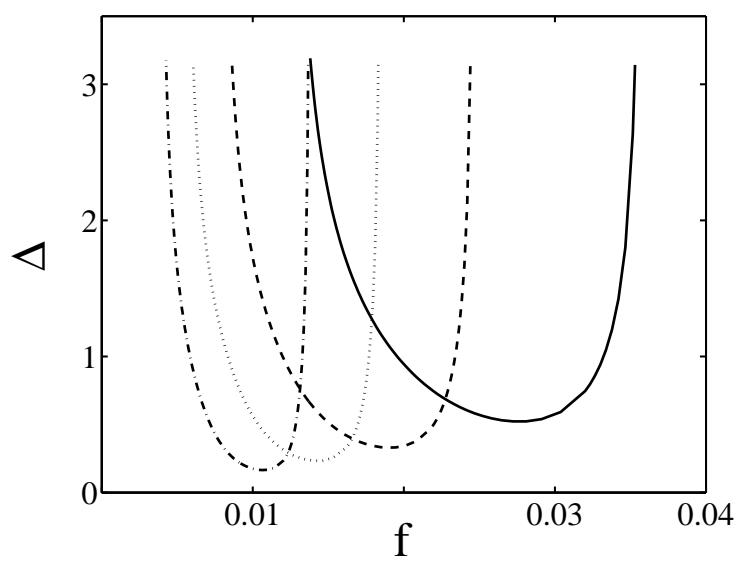

FIG. 3: The critical aspect ratio $\Delta_{1}$ for the symmetrybreaking instability as a function of $f$ for different values of $\eta$. For a fixed $\eta$, the strip state is stable below the respective curve and unstable above the curve. The parameters are: $\eta_{1}=10^{4}$ (solid line), $\eta_{2}=2.5 \cdot 10^{4}$ (dashed line), $\eta_{3}=5 \cdot 10^{4}$ (dotted line) and $\eta_{4}=10^{5}$ (dash-dot line).

To what extent is the symmetry-breaking instability sensitive to the precise form of the constitutive relations? We compared the marginal stability curves $\Delta=\Delta_{1}(f)$ for different values of $\eta$ with the respective curves 17 found for the constitutive relations of Grossman et al. [10]. A typical example of this comparison is shown in Fig. 4. One can see that, qualitatively, the results are the same: the both curves describe a symmetry-breaking instability at a critical value of the aspect ratio that depends on the area fraction. In both cases, there are sharp low- and high-density borders of instability region. Therefore, we can conclude that the instability is robust and does not require a very special form of the constitutive relations. On the other hand, there is a noticeable (about 15\%) difference in the exact positions of the marginal stability curves, so the instability provides a good quantitative test for constitutive relations of granular hydrodynamics.

\section{MORE RESULTS ON MARGINAL STABILITY}

In this Section we investigate the marginal stability problem in more detail and obtain some analytic results in the dilute limit.

\section{A. Localization and universality}

Let us characterize the strip state by the scaled parameters $\eta$ and $Z_{0}$ and introduce a different rescaling of the

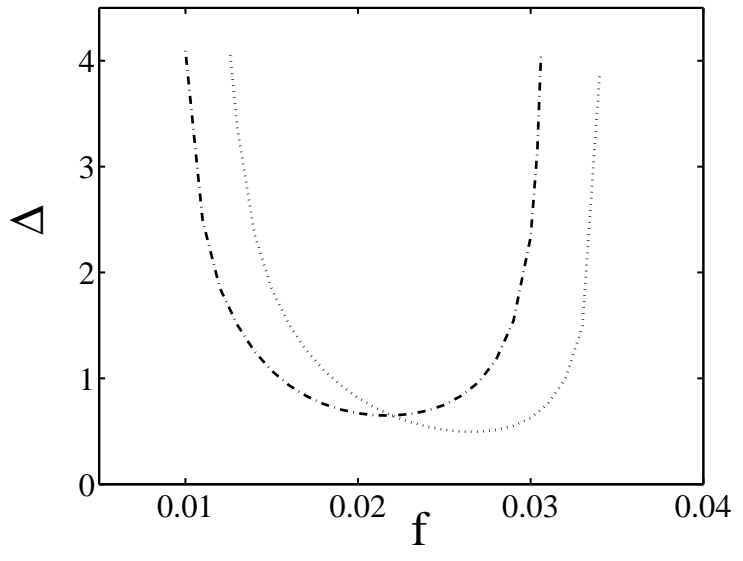

FIG. 4: The critical aspect ratio $\Delta_{1}(f)$ for the symmetrybreaking instability as computed for the constitutive relations of Grossman et al. 10 (dash-dotted line) and of JR 20] (dotted line). Parameter $\eta=11,094$.

coordinate: $\bar{x}=x \eta^{1 / 2}$. In terms of the original, physical coordinate $x_{p h}$ the new rescaling is independent of the system size:

$$
\bar{x}=\left(\frac{2 \pi}{3}\right)^{1 / 2} \frac{(1-r)^{1 / 2} x_{p h}}{d} .
$$

Equations (5) for the strip state become

$$
\begin{aligned}
\left(F Z^{\prime}\right)^{\prime}=Q, & Z(\bar{x}=0)=Z_{0} \\
\text { and } \quad Z^{\prime}(\bar{x}=0) & =0,
\end{aligned}
$$

while the eigenvalue problem (6) and (7) reads

$$
\begin{array}{r}
\phi^{\prime \prime}-\left(\frac{Q_{Z}}{F}+\bar{k}^{2}\right) \phi=0, \quad \phi^{\prime}(\bar{x}=0)=0 \\
\text { and } \quad \phi\left(\bar{x}=\eta^{1 / 2}\right)=0 .
\end{array}
$$

Now the primes denote the derivatives with respect to $\bar{x}$, while $\bar{k}=k \eta^{-1 / 2}$ is the new scaled wave number. Like the coordinate $\bar{x}$, the new scaled wave number $\bar{k}$ is independent of $L_{x}$ :

$$
\bar{k}=\left(\frac{3}{2 \pi}\right)^{1 / 2} \frac{k_{p h} d}{(1-r)^{1 / 2}},
$$

where $k_{p h}$ is the physical wave number. The problem (9) and (10) is determined by two parameters: $Z_{0}$ and $\eta$. However, $\eta$ enters the rescaled equations only in one place: in the last boundary condition in Eq. (10) where it determines the scaled system size. If the wave function $\phi(\bar{x})$ is strongly localized in the potential well $U(\bar{x})$ (correspondingly, the negative energy level is sufficiently deep), the results for $\bar{k}$ and $\phi(\bar{x})$ become independent of $\eta$ at sufficiently large $\eta$. Indeed, in this case one can safely move the boundary $\bar{x}=\eta^{1 / 2}$ to infinity. It is important that, in this case, the exact form of the boundary condition at the driving wall becomes insignificant, leading 
only to exponentially small corrections [21. This universal "localization regime" was discovered in Ref. [17] that employed a different set of constitutive relations. The criterion for localization can be obtained from the requirement that the localization length (which is of order $\bar{k}^{-1}$ ) be much smaller than the (scaled) system size $\eta^{1 / 2}$. In the physical units it corresponds to the short-wavelength limit of the bifurcating solution: $k_{p h} L_{x} \gg 1$. To fulfill this criterion, $\bar{k}$ should be far enough from the borders of the instability interval, where $\bar{k}$ vanishes. In the next subsection we will work in the dilute limit and rewrite this criterion in terms of the governing parameters of the problem.

Figure shows the marginal stability curves $\bar{k}=\bar{k}\left(Z_{0}\right)$ for different values of $\eta$, obtained numerically. Instead of the borders $f_{1}(\eta)$ and $f_{2}(\eta)$ of the instability interval in terms of parameter $f$, the respective borders in terms of parameter $Z_{0}$ appear. One can see that, for large values of $\eta$, the marginal stability curves coincide in a wide region of $Z_{0}$ not too close to the borders of the instability interval. This region corresponds to strong localization. Figures $6-8$ show the form of the potential (8) and the negative energy level $-k^{2}$ in three characteristic cases (in these figures we returned to the rescaling of the coordinates and wavenumber by the system size $L_{x}$ ). Figure 6 corresponds to the region of parameters where the energy level is deep and eigenfunction is localized. Figures 7 and 8 correspond to the parameter regions close to the low- and high-density borders of the instability, respectively. There is no localization here. Notice the qualitative change in the form of the potential near the high-density stability border. Figure 9 shows the respective eigenfunctions in these three cases.

\section{B. Marginal stability borders and physics of the instability}

The low- and high-density stability borders $f_{1}(\eta)$ and $f_{2}(\eta)$ (or respective borders in terms of $Z_{0}$ ) are determined by the zero-eigenvalue $(\bar{k}=0)$ solution of Eq. (10). This solution can be found if we know the strip solution $Z\left(\bar{x} ; Z_{0}\right)$ of Eq. (9). Indeed, it is easy to check that function $\phi_{0}(\bar{x})=F \partial Z / \partial Z_{0}$ is a solution of Eq. (10) with $\bar{k}=0$, satisfying the boundary condition $\phi^{\prime}(\bar{x}=0)$. Employing the second boundary condition $\phi\left(\bar{x}=\eta^{1 / 2}\right)=0$ we obtain $\partial Z_{1} / \partial Z_{0}=0$, where $Z_{1}=Z\left(\bar{x}=\eta^{1 / 2} ; Z_{0}\right)$. For a given $\eta$, this equation is an algebraic equation for $Z_{0}$. Our numerical results imply that this equation has only two solutions corresponding to the low- and highdensity instability borders. The instability borders have a clear physical meaning which sheds light on the physics of the instability. Let us consider the granular pressure $p=n T(1+2 G)$ 20] of the strip state, and introduce a

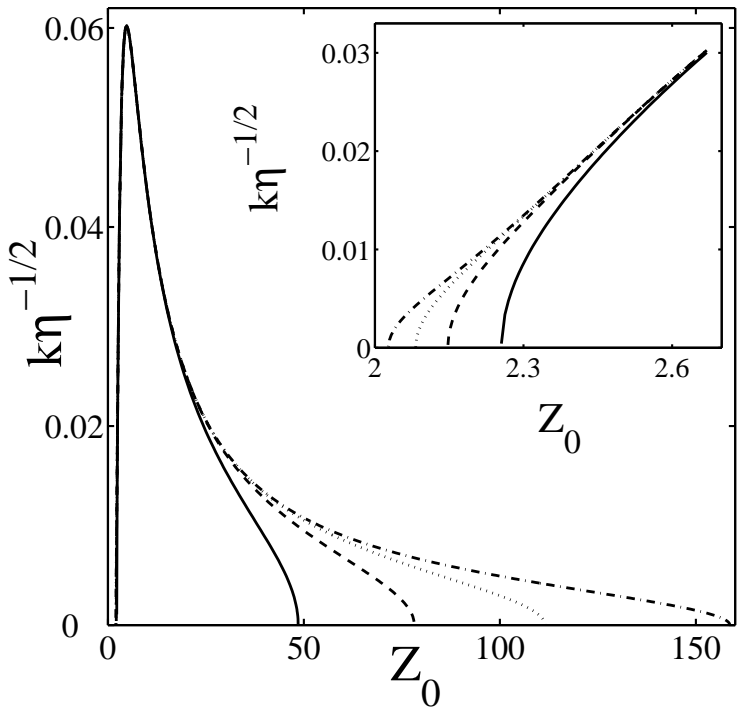

FIG. 5: Marginal stability curves for different values of $\eta$, plotted in coordinates $\bar{k}=k \eta^{-1 / 2}$ versus $Z_{0}$. For a fixed $\eta$ the strip state is stable above the respective curve and unstable below the curve. The inset shows the splitting of the curves near the high-density stability border. The values of $\eta$ are: $10^{4}$ (solid line), $2.5 \cdot 10^{4}$ (dashed line), $5 \cdot 10^{4}$ (dotted line) and $10^{5}$ (dash-dot line).

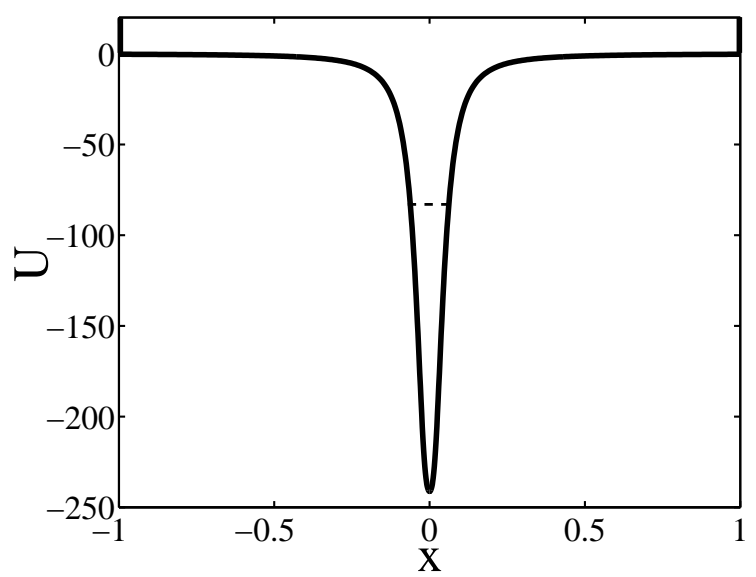

FIG. 6: An example of the negative energy level $E=-k^{2} \simeq$ -83.01 (dashed line) in the potential $U(x)$ (solid line) in the regime of localization. The parameters are $\eta=2.5 \cdot 10^{4}$ and $Z_{0}=6$.

scaled pressure

$$
P=\frac{p}{n_{c} T_{0}}=\frac{1+2 G}{Z} \frac{T}{T_{0}}
$$

As $P$ is independent of the coordinates, we can compute it at the thermal wall $\bar{x}=\eta^{1 / 2}$. Here $T=T_{0}$ and $Z=Z_{1}$, so we arrive at

$$
P=\frac{1+2 G\left(Z_{1}\right)}{Z_{1}}=P(\eta, f)
$$




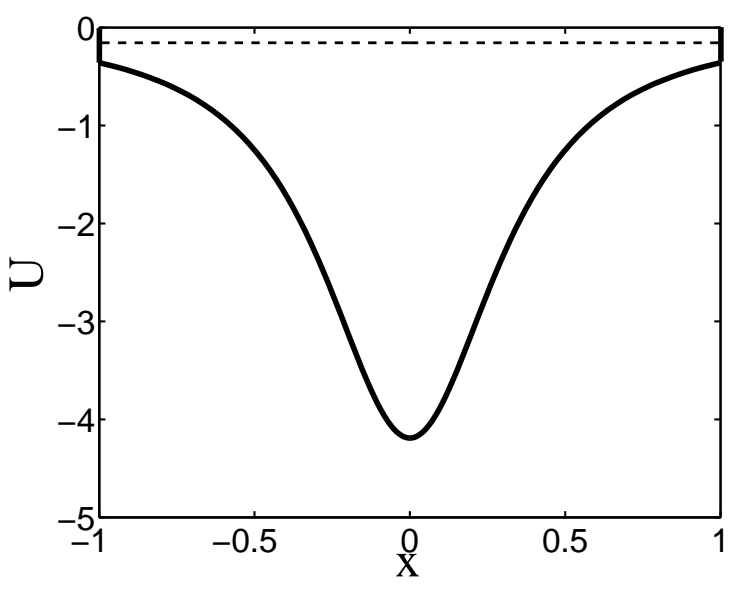

FIG. 7: An example of the negative energy level $E=-k^{2} \simeq$ -0.155 (dashed line) in the potential $U(x)$ (solid line) in the absence of localization. The parameters $\eta=2.5 \cdot 10^{4}$ and $Z_{0}=75$ correspond to the region close to the low-density stability border.

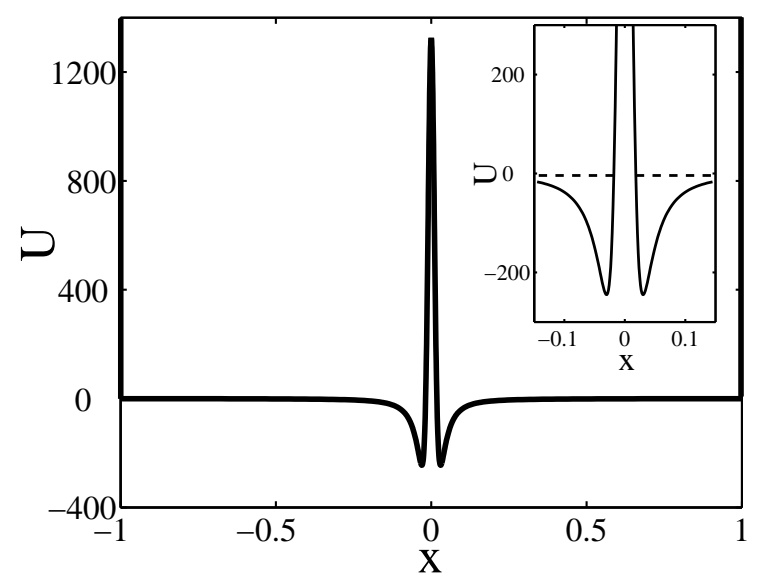

FIG. 8: Another example of the negative energy level $E=$ $-k^{2} \simeq-4.1$ (dashed line) in the potential $U(x)$ (solid line) in the absence of localization. The parameters $\eta=2.5 \cdot 10^{4}$ and $Z_{0}=2.3$ correspond to the region close to the high-density stability border.

Now let us compute the derivative $\partial P / \partial f$ at a constant $\eta$ :

$$
\frac{\partial P}{\partial f}=\frac{\partial P}{\partial Z_{1}} \frac{\partial Z_{1}}{\partial Z_{0}} \frac{\partial Z_{0}}{\partial f}
$$

One can easily check that the first and third multipliers in the right hand side of this relation are always negative. Therefore, the sign of $\partial P / \partial f$ is determined by the sign of $\partial Z_{1} / \partial Z_{0}$. As we have seen, the marginal stability borders are determined by equation $\partial Z_{1} / \partial Z_{0}=0$. Therefore, the steady-state pressure has its extremum points points exactly at the points $f_{1}$ and $f_{2}$. Figure 10 shows an example of the dependence $P=P(f)$ at a constant $\eta$ for the strip state, found numerically. One can see, that $P$ is a decreasing function of $f$ within the instability interval $\left(f_{1}, f_{2}\right)$, and an increasing function of $f$ outside

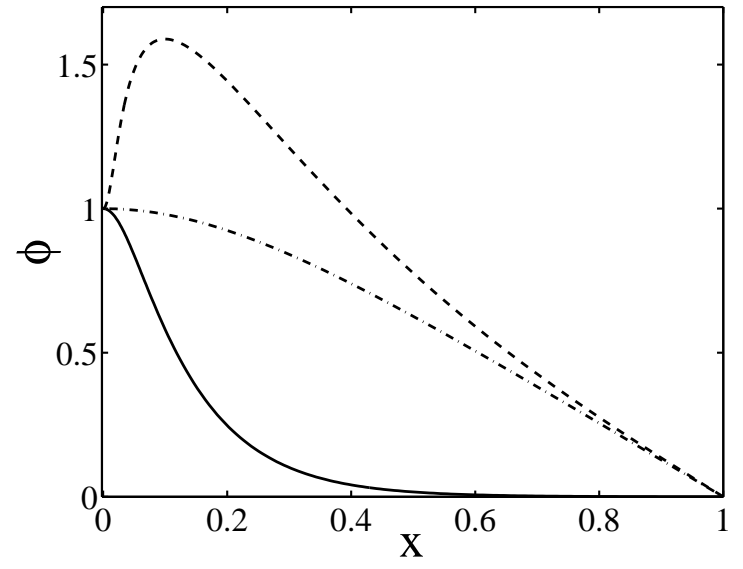

FIG. 9: Eigenfunctions $\phi(x)$ corresponding to the eigenvalues shown in Figs. 6 (strong localization, solid line), (dash-dot line) and 8 (dashed line). The eigenfunctions are (arbitrarily) normalized so that $\phi(0)=1$.

the interval. The physical interpretation of these results is clear. The presence of the anomalous (falling) part of the $P(f)$ curve indicates instability, and it is caused by the destabilizing role of collisional heat losses [ 5,12 . We can say that, on the interval $\left(f_{1}, f_{2}\right)$, the granulate has negative lateral compressibility. At $f<f_{1}$ the heat losses are too small to cause instability. The presence of the high-density border $f_{2}$ is caused by the finite-density corrections to the constitutive relations (that is, by the finite size of the particles). This is in contrast to radiative condensations in gases and plasmas, where such a stabilizing effect would be absent. Now consider a small density modulation of the strip state with a very long wavelength: $k \rightarrow 0$. For this perturbation, the stabilizing effect of the lateral heat conduction vanishes, and the negative compressibility makes the strip state unstable on the interval $\left(f_{1}, f_{2}\right)$. For a non-zero $k$, the lateral heat conduction has a stabilizing effect. Therefore, a density modulation with too a short lateral wavelength should be stable, as Fig. 2 indeed shows.

\section{Dilute limit}

In this subsection we shall work in the dilute limit and derive several analytic results. We shall see that, at large $\eta$, the dilute limit faithfully reproduces the low-density parts of the marginal stability curves.

\section{Strip state and marginal stability problem}

In the dilute limit, $Z \gg 1$, Eq. (9) for the strip state becomes

$$
\left(Z^{3 / 2}\right)^{\prime \prime}=3 Z^{-1 / 2}
$$




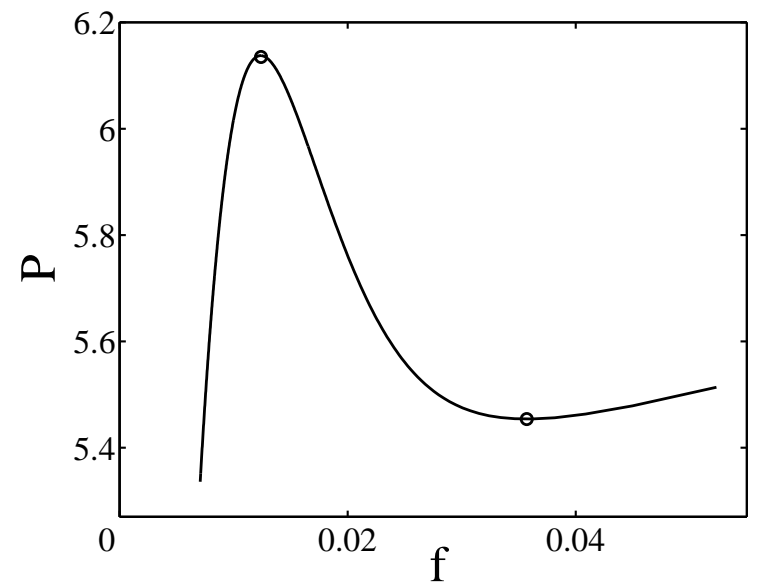

FIG. 10: The scaled steady-state granular pressure $P$ versus the grain area fraction $f$ for the strip state. The two circles correspond to the marginal stability borders $f_{1}$ and $f_{2}$. The effective lateral compressibility of the gas is negative on the interval $\left(f_{1}, f_{2}\right)$. Parameter $\eta=10^{4}$.

where the primes denote the $\bar{x}$ derivatives. The boundary conditions are $Z^{\prime}(\bar{x}=0)=0$ and $Z(\bar{x}=0)=Z_{0}$, where $Z_{0}$ is related to $f$ and $\eta$ by the normalization condition $\int_{0}^{\sqrt{\eta}} Z^{-1} d \bar{x}=f \eta^{1 / 2}$. The solution of this problem is elementary:

$$
\bar{x}=\frac{Z_{0}}{2}\left(\operatorname{arccosh} \sqrt{\zeta}+\sqrt{\zeta^{2}-\zeta}\right),
$$

where $\zeta=Z / Z_{0}$ and

$$
Z_{0}=\frac{2 \eta^{1 / 2}}{f \eta^{1 / 2}+\frac{1}{2} \sinh \left(2 f \eta^{1 / 2}\right)}
$$

(Returning for a moment to the old rescaling of the coordinate, $x_{p h} / L_{x} \rightarrow x$, one can see that the density profile (12) is determined by a single parameter: $\xi=f \eta^{1 / 2}$.) Eq. (10) for $\phi(\bar{x})=(\sqrt{3} / 2) Z^{1 / 2}(\bar{x}) \psi_{k}(\bar{x})$ takes the form

$$
\phi^{\prime \prime}-\left(\kappa-\frac{1}{\zeta^{2}}\right) \phi=0,
$$

where $\kappa=\bar{k}^{2} Z_{0}^{2}$, and $\zeta=\zeta(\bar{x})$ is given, in an implicit form, by Eq. (12). The boundary conditions for Eq. (14) remain the same as in Eq. (10). As $\zeta(\bar{x})$ is a monotonic function of $\bar{x}$, we can change the independent variable in Eq. (14) from $\bar{x}$ to $\zeta$. The resulting equation for $\phi(\zeta)$ is

$$
4(\zeta-1) \zeta \phi^{\prime \prime}+2 \phi^{\prime}+\left(1-\kappa \zeta^{2}\right) \phi=0,
$$

where the primes now denote the $\zeta$ derivatives. The function $\phi(\zeta)$ is defined on the interval $1 \leq \zeta \leq \zeta_{1}$, where $\zeta_{1}=Z_{1} / Z_{0}=\cosh ^{2} \xi$. One boundary condition is $\phi\left(\zeta=\zeta_{1}\right)=0$ to which we may add an arbitrary normalization condition $\phi(\zeta=1)=1$. An additional boundary condition, $\phi^{\prime}(\zeta=1)=(\kappa-1) / 2$, can be found from Eq. (15) itself, after substituting there $\zeta=1$. This eigenvalue problem includes a single parameter $\xi$, while $\kappa$ serves as the eigenvalue.

We have been unable to solve Eq. (15) analytically for a general $\kappa$. Still, several important asymptotics can be obtained.

\section{Zero-energy state and stability border $f_{1}$}

For $\bar{k}=0$ Eq. (15) can be solved analytically:

$$
\phi(\zeta, \bar{k}=0) \equiv \phi_{0}(\zeta)=\sqrt{\zeta}-\sqrt{\zeta-1} \operatorname{arccosh} \sqrt{\zeta},
$$

In other words, we impose a zero eigenvalue $\bar{k}=0$ and find the low-density stability border $f_{1}=f_{1}(\eta)$ from the boundary condition $\phi_{0}\left(\zeta_{1}\right)=0$. We obtain an algebraic equation $\operatorname{coth}\left(\xi_{1}\right)=\xi_{1}$ for $\xi_{1}=f_{1} \eta^{1 / 2}$. Its solution is $\xi_{1}=1.19968 \ldots$. This result explains why all marginal stability curves shown in Fig. 2 depart (almost) from the same point at the low-density side. Figure 11 compares the scaled quantity $f_{1} \eta^{1 / 2}$ at different $\eta$, found numerically from Eq. (6), with this analytic prediction (a constant). The agreement is very good for large $\eta$. As $\eta$ goes down, $f_{1}$ increases and the dilute approximation starts to deteriorate.

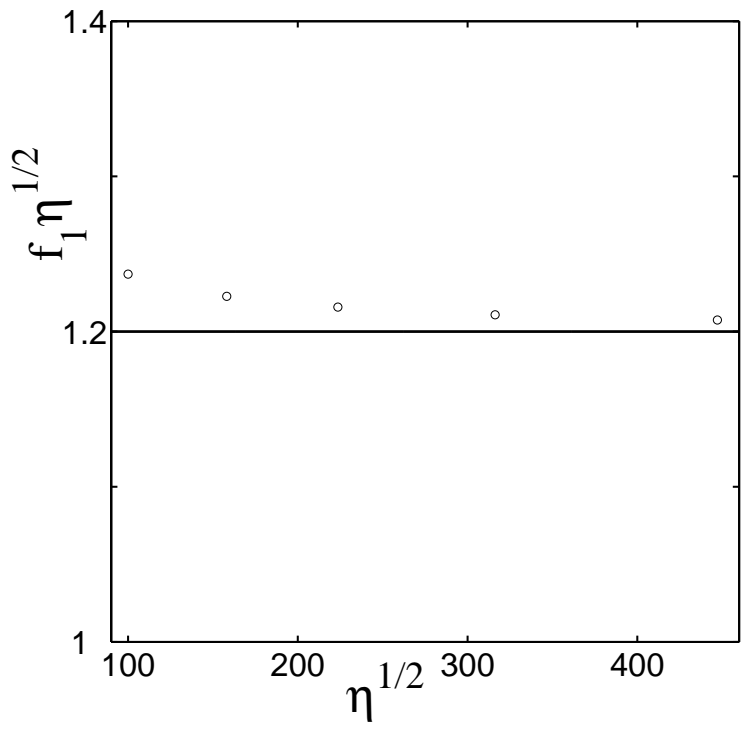

FIG. 11: The scaled low-density stability boundary $\xi_{1}=$ $f_{1} \eta^{1 / 2}$ at different $\eta^{1 / 2}$ as found numerically from Eq. (6) (circles). Solid line shows the analytical result $\xi_{1}=1.19968 \ldots$ obtained in the dilute limit.

Figure 12 compares the analytic result for the zeroenergy eigenfunction $\phi_{0}(x)$, given by Eqs. (16) and (12), with a numerical solution of Eq. (6) for $\eta=10^{4}$. The coordinate $x$ in Fig. 12 is rescaled by $L_{x}$. The analytic and numeric results are obtained for slightly different values of $f_{1}$ (see Fig. 11). One can see that the agreement is excellent. 


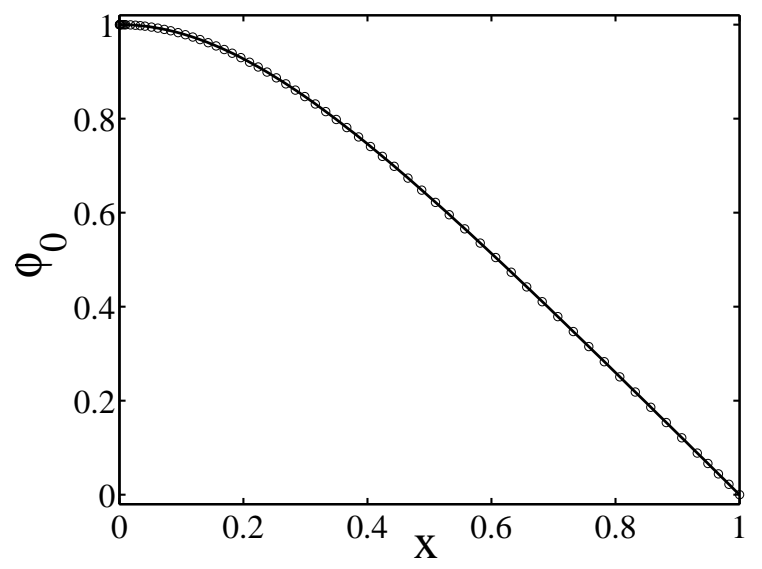

FIG. 12: The zero-energy eigenfunction $\phi_{0}(x)$ computed numerically from Eq. (6) (circles) and given analytically by Eqs.(16) and (12) (solid line). The parameters are $\eta=10^{4}$, $f_{1, \text { num }}=0.0124$ (circles) and $f_{1, \text { anal }}=0.0120$ (solid line).

\section{Short-wavelength limit: localization and universality}

In the short-wavelength limit the system boundary $\zeta=\zeta_{1}=\cosh ^{2} \xi$ can be moved to infinity. This requires a strong inequality $\xi=f \eta^{1 / 2} \gg 1$. In this limit, the eigenvalue problem (15) does not include any parameter. The eigenvalue $\kappa$ should therefore be a number of order of unity, hence $\bar{k}=A / Z_{0}$, with constant $A$ of order of unity. The constant can be found numerically: $A \simeq 0.525$. This simple result represents the low-density limit of the "universal" marginal stability curve, corresponding to strong localization. Figure 13 shows this asymptotics for $\eta=10^{5}$. One can see excellent agreement for large enough $Z_{0}$, but not too close to the higher- $Z_{0}$ (low-density) instability border. Near the instability border $\xi$ becomes of order of unity, and localization breaks down.

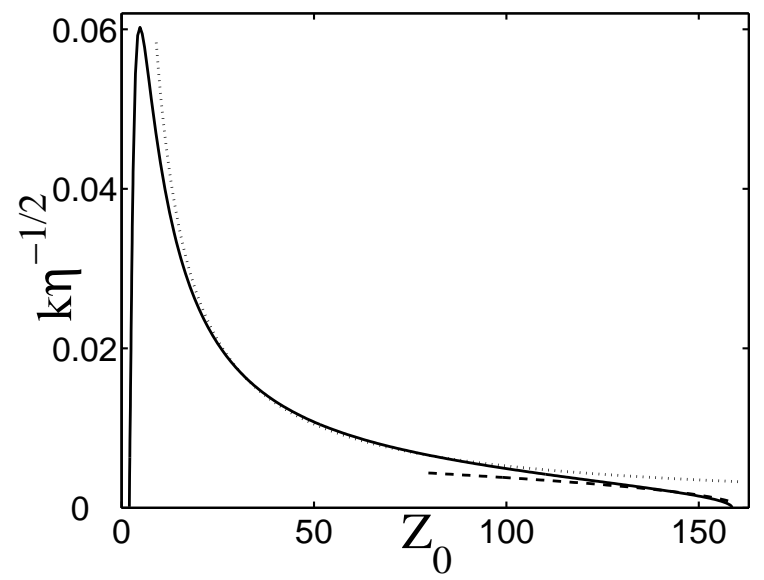

FIG. 13: The marginal stability curve for $\eta=10^{5}$, plotted in coordinates $\bar{k}=k \eta^{-1 / 2}$ versus $Z_{0}$ (solid line) and two dilute-limit asymptotics: the dilute-limit part $\bar{k}=A / Z_{0}$ of the ("universal") short-wavelength curve (dotted line) and the long-wavelength asymptotics 24) (dashed line).
Returning to the parameters $\eta$ and $f$, and to the wave number $k=k_{p h} L_{x}$, we can rewrite the asymptotics $\bar{k}=$ $A / Z_{0}$ as

$$
k=\frac{A}{2}\left[f \eta^{1 / 2}+\frac{1}{2} \sinh \left(2 f \eta^{1 / 2}\right)\right] .
$$

This asymptotics is shown in Fig. 14.

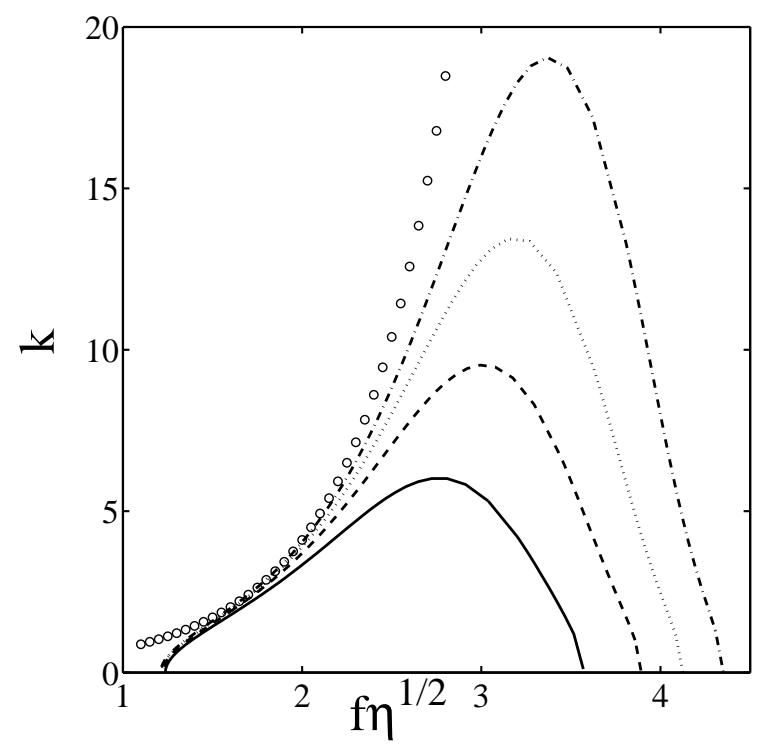

FIG. 14: Marginal stability curves for different values of $\eta$, plotted in coordinates $k$ versus $\xi=f \eta^{1 / 2}$. For a fixed $\eta$, the strip state is stable above the respective curve and unstable below the curve. The values of $\eta$ are: $10^{4}$ (solid line), $2.5 \cdot 10^{4}$ (dashed line), $5 \cdot 10^{4}$ (dotted line) and $10^{5}$ (dash-dot line). Also shown is the dilute-limit asymptotics (17) of the universal marginal stability curve.

The asymptotics (17) is valid when $\xi=f \eta^{1 / 2} \gg 1$. It is easy to check that this criterion coincides, in the dilute limit, with the localization criterion $\bar{k}^{-1} \ll \eta^{1 / 2}$ discussed in Sec. 4A. On the other hand, the parameter $\xi$ should not be too large, so that the dilute limit condition $Z_{0} \gg 1$ is still satisfied, see Eq. (13). These two criteria can be rewritten as a strong double inequality for $f$ :

$$
\frac{1}{\eta^{1 / 2}} \ll f \ll \frac{\log \left(8 \eta^{1 / 2}\right)}{2 \eta^{1 / 2}},
$$

that can be satisfied only for extremely large $\eta$.

\section{Long-wavelength limit: perturbation theory}

Close to the low-density stability border, $f-f_{1} \ll$ $f_{1}=1.19968 \ldots \eta^{-1 / 2}$ we can assume that $\kappa \ll 1$ and solve Eq. (15) perturbatively. In the physical units, this strong inequality corresponds to the long-wavelength limit: $k_{p h} L_{x} \ll 1$. In its turn, the dilute limit requires $\eta \gg 1$. We substitute in Eq. (15) $\phi(\zeta)=\phi_{0}(\zeta)+\kappa \Omega(\zeta)$, 
where $\phi_{0}$ is given by Eq. (16). Neglecting the $\kappa^{2}$-term in Eq. (15), we obtain

$$
4(\zeta-1) \zeta \Omega^{\prime \prime}+2 \Omega^{\prime}+\Omega=\zeta^{2} \phi_{0}(\zeta),
$$

The normalization and boundary conditions at $\zeta=1$ are $\Omega(\zeta=1)=0$ and $\Omega^{\prime}(\zeta=1)=1 / 2$, respectively. The latter condition follows from Eq. (18) itself. Equation (18) can be solved analytically. With the account of the two boundary conditions we obtain

$$
\Omega(\zeta)=-\frac{1}{8} \Phi_{2}(\zeta)+\Phi_{2}(\zeta) I_{1}(\zeta)-\Phi_{1}(\zeta) I_{2}(\zeta),
$$

where $\Phi_{1}(\zeta)=(\zeta-1)^{1 / 2}$ and $\Phi_{2}(\zeta)=-2 \phi_{0}(\zeta) . I_{1}$ and $I_{2}$ are indefinite integrals:

$$
I_{1}=\int^{\zeta} \frac{\Phi_{1} G_{1}}{G_{2} W} d \zeta \quad \text { and } \quad I_{2}=\int^{\zeta} \frac{\Phi_{2} G_{1}}{G_{2} W} d \zeta,
$$

where $G_{1}=\zeta^{2} \phi_{0} / 4, G_{2}=\zeta(\zeta-1), W=\Phi_{1} \Phi_{2}^{\prime}-\Phi_{1}^{\prime} \Phi_{2}$ and the primes denote the $\zeta$ derivatives. Integrals $I_{1}$ and $I_{2}$ can be evaluated in elementary functions, but the results are too cumbersome to be presented here. The additional boundary condition [at $\zeta=Z_{1} / Z_{0} \equiv \zeta_{1}$ ] reads $\phi\left(\zeta_{1}\right)=\phi_{0}\left(\zeta_{1}\right)+\kappa \Omega\left(\zeta_{1}\right)=0$ which yields

$$
\kappa(\xi)=-\phi_{0}\left(\zeta_{1}\right) / \Omega\left(\zeta_{1}\right),
$$

where $\zeta_{1}=\cosh ^{2} \xi$. At the low-density stability border $f=f_{1}$ we have $\xi=\xi_{1}=1.19968 \ldots$. In the perturbative treatment, one should expand $\kappa(\xi)$ near $\xi=\xi_{1}$ up to the linear term $\xi-\xi_{1}$. The zero-order term vanishes, and we obtain

$$
\kappa(\xi)=-\frac{\Phi_{2}^{\prime}\left(\xi_{1}\right)}{2 \Phi_{1}\left(\xi_{1}\right) I_{2}\left(\xi_{1}\right)}\left(\xi-\xi_{1}\right) .
$$

In the physical variables we have

$$
k_{p h} L_{x}=\frac{\eta^{1 / 2}}{Z_{0}\left(\xi_{1}\right)}\left(-\frac{\Phi_{2}^{\prime}\left(\xi_{1}\right)}{2 \Phi_{1}\left(\xi_{1}\right) I_{2}\left(\xi_{1}\right)}\right)^{1 / 2}\left(\xi-\xi_{1}\right)^{1 / 2} .
$$

As $Z_{0}$ is proportional to $\eta^{1 / 2}$, the right hand side of Eq. (22) is actually independent of $\eta$. Evaluating the integral $I_{2}\left(\xi_{1}\right)$, we obtain $I_{2}\left(\xi_{1}\right)=-0.883381 \ldots$. The final result is

$$
k_{p h} L_{x}=2.5115 \ldots\left(\xi-\xi_{1}\right)^{1 / 2} .
$$

Alternatively, we obtain

$$
\begin{aligned}
\bar{k}\left(Z_{0}\right) & =\left(\frac{\xi_{1}}{Z_{0}\left(\xi_{1}\right)}\right)^{3 / 2}\left(\frac{Z_{0}-Z_{0}\left(\xi_{1}\right)}{2 I_{2}\left(\xi_{1}\right)}\right)^{1 / 2} \\
& =0.000485 \ldots\left(Z_{0}\left(\xi_{1}\right)-Z_{0}\right)^{1 / 2} .
\end{aligned}
$$

The asymptotics (24) is depicted in Fig. 13. Close to the higher- $Z_{0}$ (low-density) stability border it shows good agreement with the marginal stability curve found numerically.

\section{SUMMARY AND DISCUSSION}

We determined the criteria for the spontaneous symmetry-breaking instability of the laterally uniform granular cluster (strip state) in a prototypical driven granular gas. Working in the limit of nearly elastic particle collisions and low or moderate densities, we employed granular hydrodynamics with the Jenkins-Richman constitutive relations [20]. The instability of the strip state can be interpreted in terms of negative compressibility of the granulate in the lateral direction. An important limit is found, when the marginal stability curves are independent of the details of the boundary condition at the driving wall. In this regime the density perturbation is exponentially localized at the elastic wall opposite to the driving wall. Working in the dilute limit, we obtained some analytic asymptotics of the marginal stability curves.

The results of this work show that the symmetrybreaking instability predicted in Ref. [17] is robust and does not require very special constitutive relations. The marginal stability curves obtained in this work are quite similar to those obtained earlier 17] for a different set of constitutive relations (see Fig. (1). There are some quantitative differences, however. Therefore, the instability provides a sensitive test to the accuracy of constitutive relations.

This work was focused on the criteria of instability of the strip state. In systems sufficiently long in the lateral direction, instability occurs in a whole range of wave numbers $k$ (below the respective marginal stability curve). Correspondingly, multiple steady state solutions with different $k$ are possible. In a laterally infinite system, these solutions are periodic in the lateral coordinate. A finite system selects a finite number of wavelengths 17. An important issue that was not addressed in this work is selection: what is the wavelength of the resulting symmetry-broken cluster in an infinite, or long enough, system? The selection has dynamical nature; this important issue is addressed elsewhere [22].

Recently, the predicted symmetry-breaking instability has been observed in particle simulations [23]. We hope it will be investigated in experiment, too. The experimental setting can be of the type used by Kudrolli et al. 17. 16]: a system of steel spheres, rolling on a smooth surface and driven by a rapidly vibrating side wall. The present work (see also Ref. 17]) provides the region of parameters where the instability can be observed. An important issue is to eliminate the static friction between the particles and surface that occurs far enough from the driving wall. In experiment, this is achieved by slightly inclining the system, so that a very small gravity appears 17, 16. As the result, the strip state moves down, toward the driving wall [7]. The model problem investigated in the present work does not include gravity. We expect, 
however, that the symmetry-breaking instability should persist for a non-zero gravity. In fact, a similar instability has already been observed in particle simulations of a dilute two-dimensional granular bed fluidized by a rapidly vibrating bottom plate 24. Under conditions of the simulations [24] there was no direct, mechanical coupling between the bottom plate vibration and collective granular motions. Therefore, the vibrofluidized system, investigated in Ref. 24], is similar (though not identical) to the model system driven by a thermal wall. As gravity introduces an additional scaled parameter, the phase diagram of this type of systems should be more complicated. For example, it is already known that, at some values of the scaled parameters, steady "thermal" convection (steady state of a different type) develops both in vibrofluidized systems 24, 25 and in systems driven by a "thermal" wall 26, 27]. Granular hydrodynamics will be instrumental in delineating the phase diagrams of these systems in the limit of nearly elastic collisions.

Finally, when inelasticity of the particle collisions is not small, the normal stress difference, possible lack of scale separation and non-Gaussianity in the velocity distribution may become important. The potential role of these effects in the symmetry-breaking instability should be the subject of further investigations.

\section{ACKNOWLEDGEMENTS}

We are very grateful to P.V. Sasorov and J.P. Gollub for useful discussions. This research was supported by the Israel Science Foundation, founded by the Israel Academy of Sciences and Humanities.

[1] H.M. Jaeger, S.R. Nagel, and R.P. Behringer, Rev. Mod. Phys. 68, 1259 (1996); Physics Today, April 1996, p. 32.

[2] J. Duran, Sands, Powders and Grains. An Introduction to the Physics of Granular Materials. (Springer, New York, 2000).

[3] L.P. Kadanoff, Rev. Mod. Phys 71, 435 (1999).

[4] M.A. Hopkins and M.Y. Louge, Phys. Fluids A 3, 47 (1991).
[5] I. Goldhirsch and G. Zanetti, Phys. Rev. Lett. 70, 1619 (1993).

[6] S. McNamara and W.R. Young, Phys. Rev. E 53, 5089 (1996).

[7] A. Kudrolli, M. Wolpert, and J.P. Gollub, Phys. Rev. Lett. 78, 1383 (1997).

[8] J.S. Olafsen and J.S. Urbach, Phys. Rev. Lett. 81, 4369 (1998).

[9] Y. Du, H. Li, and L.P. Kadanoff, Phys. Rev. Lett. 74, 1268 (1995).

[10] E.L. Grossman, T. Zhou, and E. Ben-Naim, Phys. Rev. E 55, 4200 (1997).

[11] S.E. Esipov and T. Pöschel, J. Stat. Phys. 86, 1385 (1997).

[12] B. Meerson, Rev. Mod. Phys. 68, 215 (1996).

[13] C.S. Campbell, Annu. Rev. Fluid Mech. 22, 57 (1990).

[14] J.J. Brey and D. Cubero, Phys. Rev. E 57, 2019 (1998).

[15] J. Tobochnik, Phys. Rev. E 60, 7137 (1999).

[16] A. Kudrolli and J. Henry, Phys. Rev. E 62, R1489-R149 (2000).

[17] E. Livne, B. Meerson, and P.V. Sasorov, Phys. Rev. E 65, 021302 (2002).

[18] J.J. Brey and D. Cubero, in Granular Gases, edited by T. Pöschel and S. Luding (Springer, Berlin, 2001), pp. 59-78; I. Goldhirsch, ibid, pp. 79-99.

[19] M.C. Cross and P.C. Hohenberg, Rev. Mod. Phys. 65, 851 (1993).

[20] J.T. Jenkins and M.W. Richman, Phys. Fluids 28, 3485 (1985).

[21] This statement is true only for a certain class of boundary conditions: those specifying either the heat flux, or the granular temperature at the boundaries. For vibrofluidized systems, the boundary conditions of this type are usually obtained under two conditions. First, the vibration amplitude of the driving plate should be much less than the mean free path of the granulate near the driving plate. Second, the vibration frequency should be much higher than the particle collision rate near the plate.

[22] E. Livne, B. Meerson and P.V. Sasorov (in preparation).

[23] T. Schwager, B. Meerson and T. Pöschel (in preparation).

[24] P. Sunthar and V. Kumaran, Phys. Rev. E 64, 041303 (2001).

[25] R.D. Wildman, J.M. Huntley, and D.J. Parker, Phys. Rev. Lett. 86, 3304 (2001).

[26] R. Ramìrez, D. Risso, and P. Cordero, Phys. Rev. Lett. 85, 1230 (2000).

[27] X. He, B. Meerson and G. Doolen, Phys. Rev. E 65, 0203XX(R) (2002). 